\title{
STUDY OF A DETUNED ACCELERATING SECTION WITH THE COMPUTER PROGRAM PROGON*
}

\author{
S. A. Heifets, S. A. Kheifets \\ Stanford Linear Accelerator Center, Stanford University, Stanford, CA 94309 USA
}

\section{tbstract}

The longitudinal coupling impedance for a number of lower passbands, bunch to bunch energy variation due to longitudinal wake fields, the beam loading compensation, some effects of production errors, and the of pulse transmission through a detuned disk loaded accelerating section with finite wall conductivity have been studied using the computer program PROGON.

\section{MOTIVATION AND METHOD}

The detuned accelerating section [1] desigaled to diuinish the undesirable effects of the transverse wake field has been studied previously using approximate methods. [2] [3] Here these results are confirmed by employing the new computer code PROGON [4] which is based on the field matching . ochnique for the Fourier harmonics of the longitudinal electromagnetic (EM) travelling waves. The geometry of the considered 204 cell section is presented in Fig. 1a.
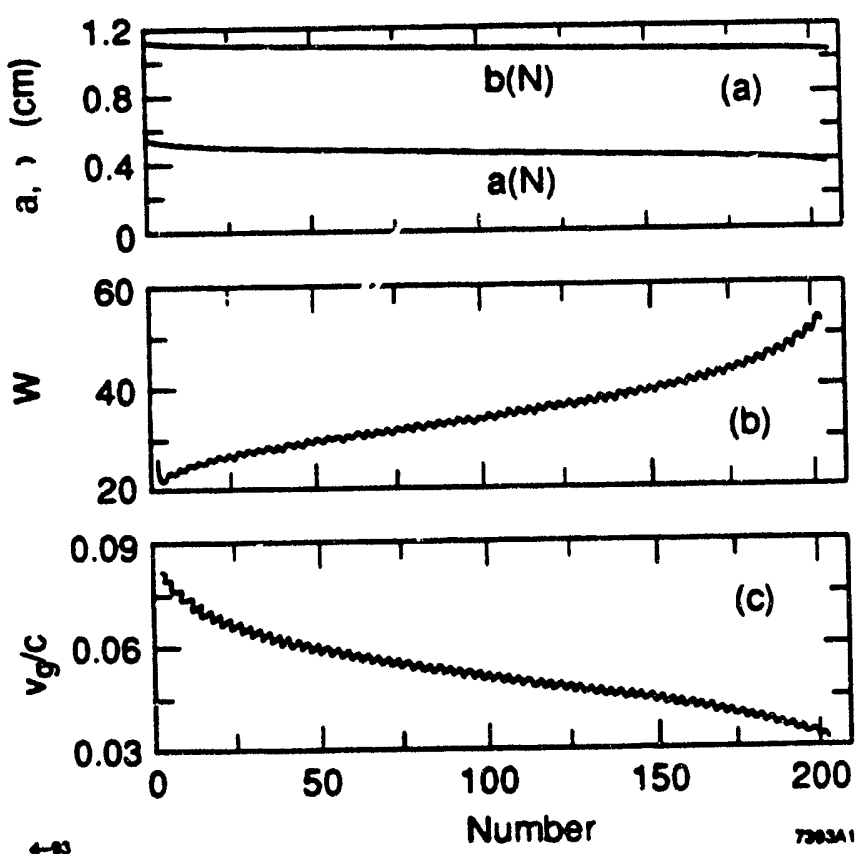

Figure 1. (a) The radii of the $N$ th cavity $b(N)$ and iris $a(N)$ for the considered 204 cell section. The gaps $g(N)=0.729 \mathrm{~cm}$, the iris thicknesses $l(N)=0.149 \mathrm{~cm}$. (b) The stored energy in the $N$ th cell $W(N)$. (c) The relative group velocity in the $N$ th cell $v_{g}(N) / c$.

* Work supported by Department of Energy contract DE-AC03-76SF00515.

\section{ACCELERATING FIELD}

First, the EMI fields in the structure, the corresponding stored energy $W(N)$ per cell and the power flow $S_{:}\left(\Lambda^{\circ}\right)$ through the cavily cross section are found. These quantities define the relative group velocity $v_{g} / c=S_{z} d / I^{\circ}$. where $d$ is the cell length. The stored energy $W$ and the group velocity $v_{\text {g }}$ are plotted in Figs. 1b,c, respectively.

\section{ERRORS AND DAMPING}

Next, the effect of fabrication errors for infinite and finite wall conductivity on the excitation of and propagation through the section of a cylindrical accelerating wave with frequency $f$ has been studied. The absolute values of the reflection $R(f)$ and the transmission $T(f)$ coefficients are plotted in Fig. 2 for 'small' relative random radii er-

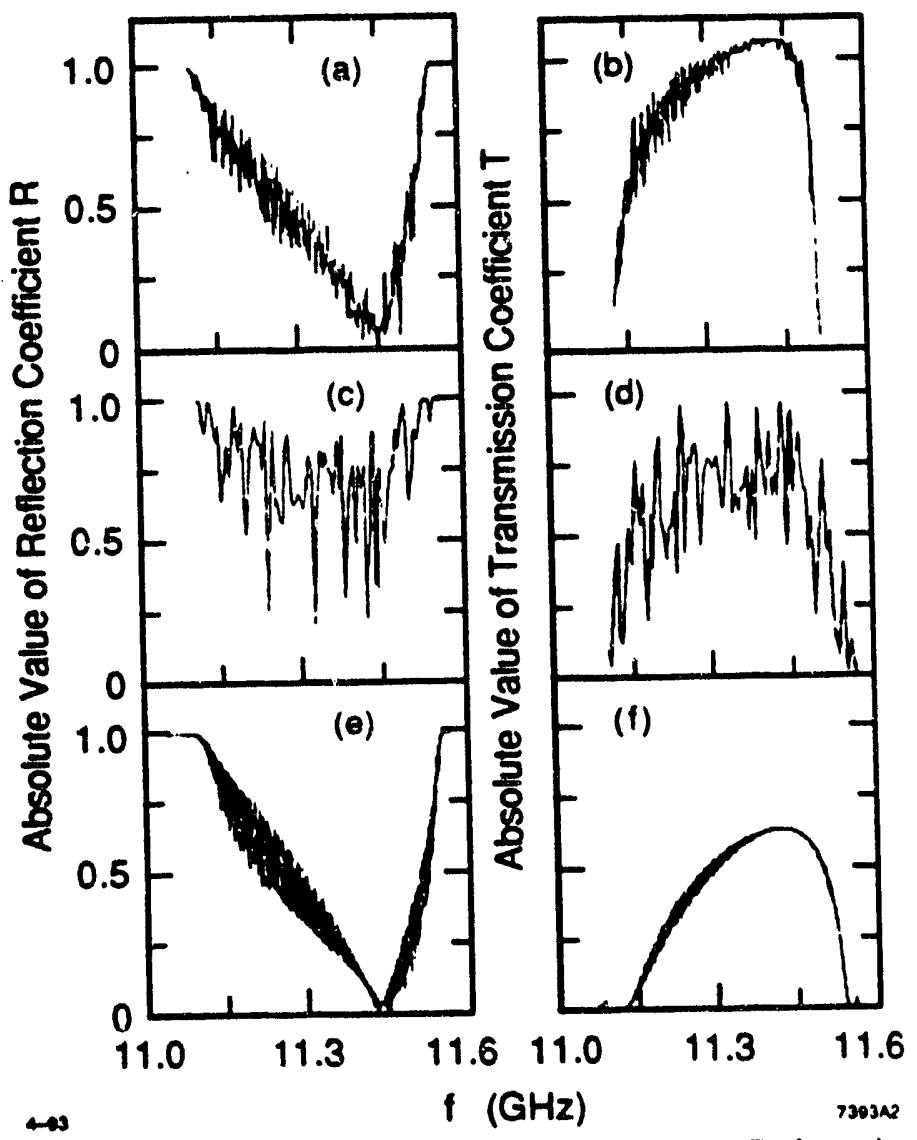

Figure 2. The ahsolute values of the reflection $R(f)$ and the transmission $T(f)$ coefficients: (a),(b) 'small' relative random radii errors in the range $\Delta a / a=\Delta b / b=$ $\pm 2.5 \cdot 10^{-4} ;$ (c).(d) large errors $\Delta a / a=\Delta b / b=$ $\pm 2.5 \cdot 10^{-3}$ : (e).(f) the finite conductivity of the copper

walls. 83 3

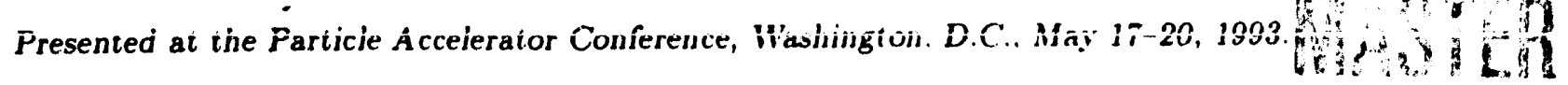




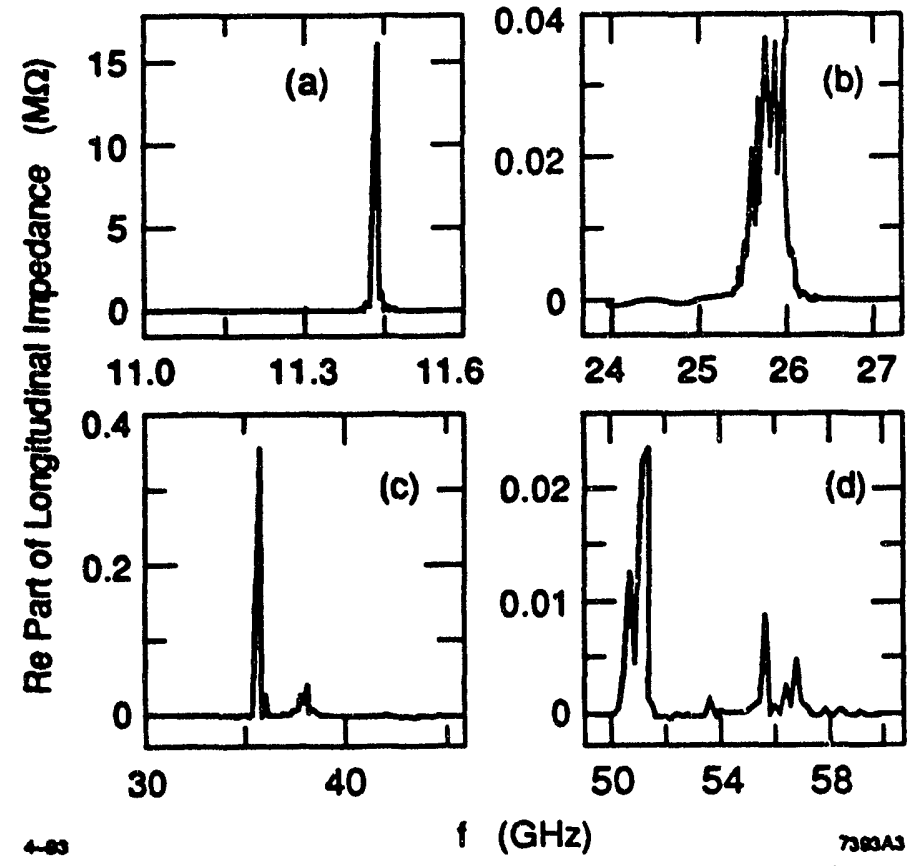

Figure 3. The real part of the longitudinal coupling impedance $Z_{c}(f)$ for a few lowest passbands.
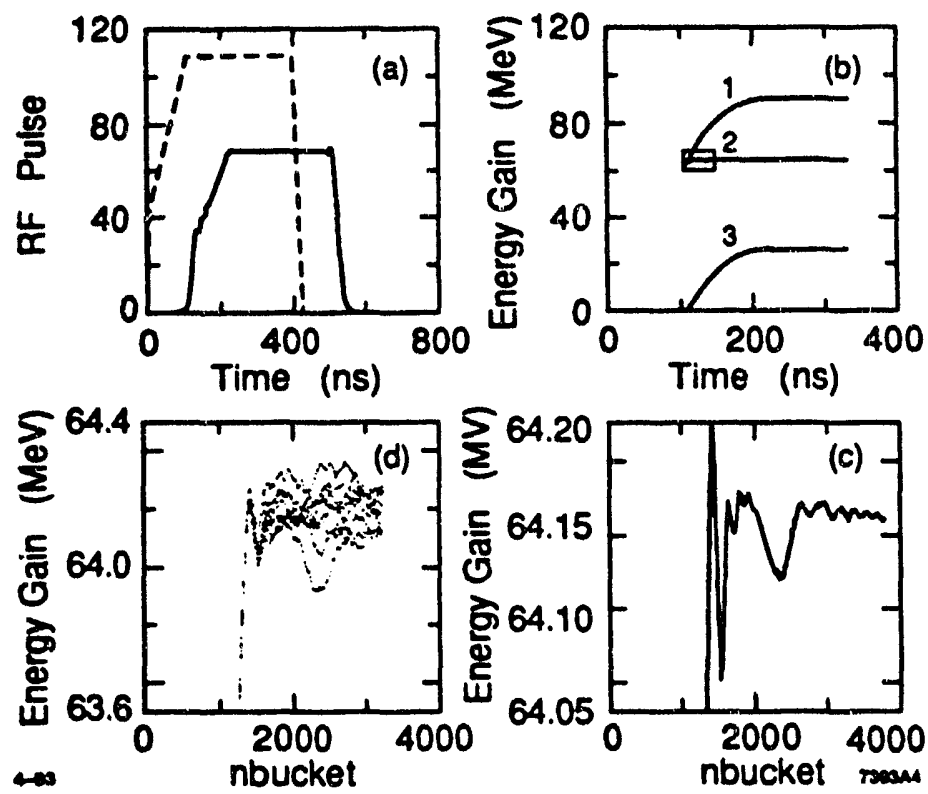

Figure 4. (a) The envelopes of the rf pulse at the entrance (dashed curve) and exit (solid curve); (b) The energy gain by a bunch in an accelerating wave with power $70.56 \mathrm{MW}$ (curve 1), the energy loss due to wake fields of the previous bunches (curve 3 ) and their difference (curve 2) versus the bunch number. The number of electrons is $N_{p}=0.7 \cdot 10^{10}$ per bunch. (c) A close up of part of curve 2 in Fig. $4 \mathrm{~b}$. (d) The effect of the random bunch population in the range $\Delta N_{p} / N_{p}= \pm 3 \%$. This plot was obtained using ten different sets of random numbers for the same conditions as in Fig. $4 b, c$. rors (Figs. 2a,b), for 'large' errors (Figs. 2c,d), and for the copper wall conductivity (Figs. $2 e, f$ ) without errors.

\section{COUPLING IMPEDANCE}

The real part of the longitudinal coupling impedance $Z_{c}(f)$ is presented in Fig. 3 for a few lower passbands.

\section{PULSE TRANSMISSION}

The distortion of an if pulse transmitted through the detuned section with finite wall conductivity is illustrated in Fig. $4 a$ where the envelopes of the if pulse at the entrance (dashed curve) and the exit (solid curve) are plotted [5].

\section{BEAM LOADING}

The beam loading [6] for a long train of bunches is illustrated in Fig. 4b [7]. The energy gain by a bunch in an accelerating wave with power $70.56 \mathrm{MW}$ (curve 1), the energy loss due to wake fields of the previous bunches (curve 3 ) and their difference (curve 2) are plotted versus the bunch number for the number of electrons $N_{p}=0.7 \cdot 10^{10}$ per bunch. The impedance of the first passband only is taken into account for the wake field calculations. Fig. $4 \mathrm{c}$ is a close up of part of curve 2 in Fig. $4 b$. Fig. $4 d$ illustrates the effect of the random bunch population in the range $\Delta N_{p} / N_{p}= \pm 3 \%$.

\section{ACKNOWLEDGEMENTS}

We are grateful to K. Bane, R. Miller, R. Ruth and J. Wang for useful discussions.

\section{REFERENCES}

1. J.W. Wang, B.W. Littmann, "Design Study on Quasi-Constant Gradient Accelerating Structure," SLAC/AP-32, 1991.

2. R. Helm, "Computation of the properties of traveling-wave linac structures," SLAC-PUB-813 (1970).

3. K. Thompson, R. Ruth "Controlling Transverse Multi-bunch Instabilities in Linacs," Phys. Review D41, 964 (1990).

4. S.A. Heifets, S.A. Kheifets "Longitudinal Electromagnetic Fields in an Aperiodic Structures," SLAC-PUB-5907, 1992.

5. S.A. Heifet3, S.A. Kheifets "RF Pulse Transmission through an Accelerating Section," SLAC Note AAS-74, September 1992.

6. R. Ruth, "Multi-bunch Energy Compensation," SLAC-PUB-4541, 1988.

7. S.A. Heifets, S.A. Kheifets "Study of the Energy Gain and the Beam Loading of the Detuned Structures with a Simple Model," SLAC/AP-96, January 1993. 

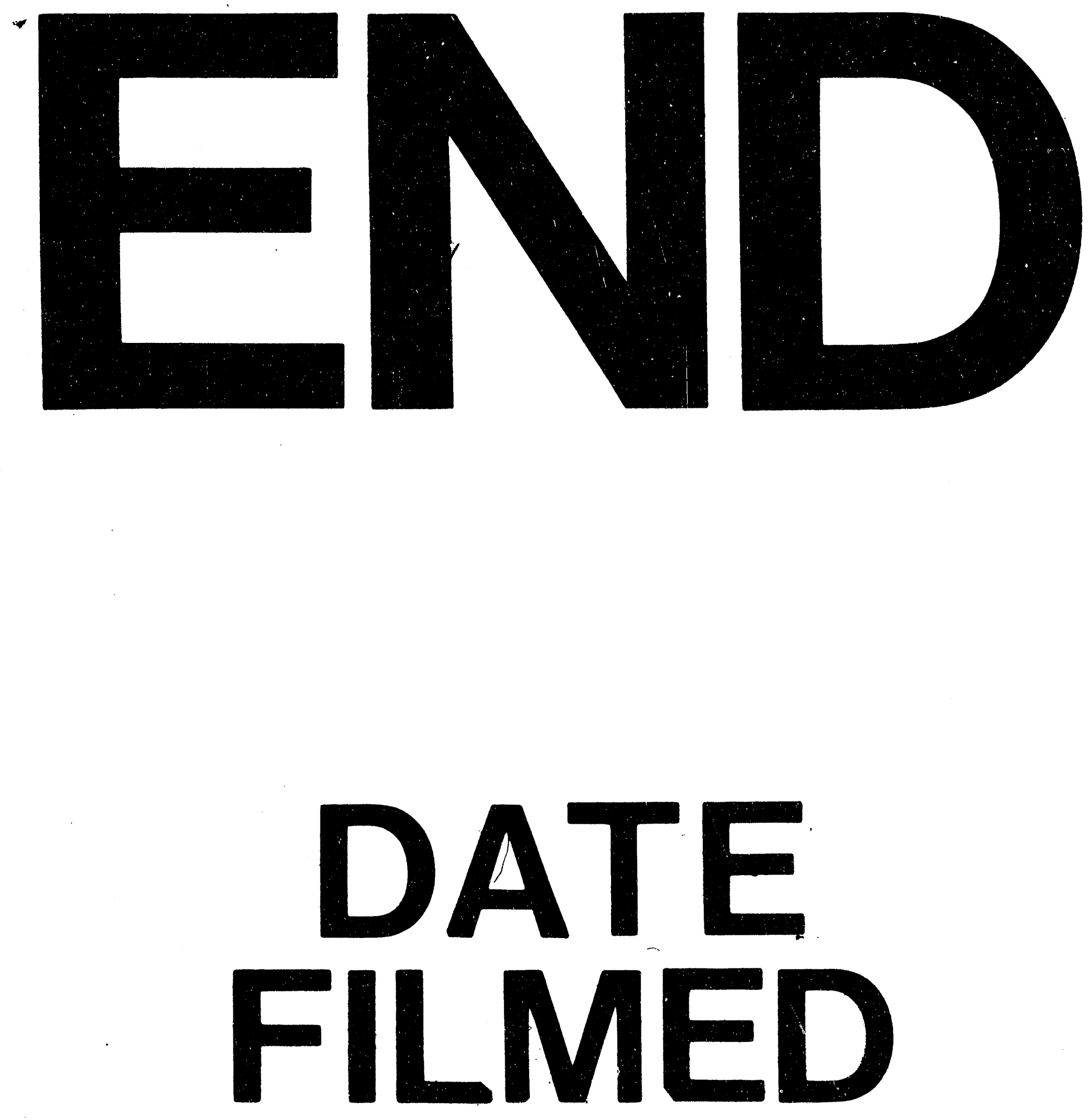

I

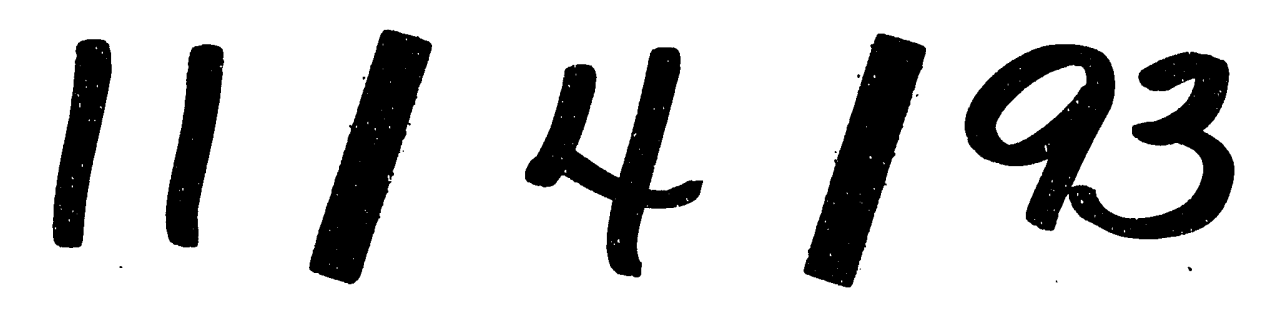


